\title{
Crystalline Lens Dislocation
}

National Cancer Institute

\section{Source}

National Cancer Institute. Crystalline Lens Dislocation. NCI Thesaurus. Code C118864.

Displacement of the crystalline lens from the visual axis. 